\title{
In vivo excision of a single targeted nucleotide from an mRNA by a trans excision-splicing ribozyme
}

\author{
DANA A. BAUM and STEPHEN M. TESTA \\ Department of Chemistry, University of Kentucky, Lexington, Kentucky 40506, USA
}

\begin{abstract}
We have previously reported the development of a group I intron-derived ribozyme that can bind an exogenous RNA substrate and excise from that substrate an internal segment in vitro, which allows for sequence-specific modification of RNA molecules. In this report, the activity of this trans excision-splicing ribozyme in a cellular environment, specifically Escherichia coli, was investigated. The ribozyme was re-engineered to target for excision a single-base insertion in the transcript of a green fluorescent protein, and fluorescence was exploited as a reporter for trans excision-splicing. We show that the ribozyme is able to catalyze the trans excision-splicing reaction in vivo and can repair the mutant transcripts. On average, $12 \%$ correction is observed as measured by fluorescence and at least $0.6 \%$ correction as confirmed through sequence analysis. This represents the first report of a biomolecule (in this case a ribozyme) that can selectively excise a targeted nucleotide from within an mRNA transcript in vivo. This new class of biochemical tools makes possible a wide variety of new experimental strategies, perhaps including a new approach to molecular-based therapeutics.
\end{abstract}

Keywords: trans excision-splicing; ribozyme; RNA repair

\section{INTRODUCTION}

Group I introns are catalytic RNAs (Kruger et al. 1982) with the ability to splice themselves out of RNA transcripts. Eliminating the $5^{\prime}$ and $3^{\prime}$ exon sequences from these self-splicing introns produces catalytic ribozymes that are able to bind substrates in trans and perform reactions analogous to the reaction steps of self-splicing (Zaug and Cech 1986; Zaug et al. 1986; Cech 1990; Sargueil and Tanner 1993; Testa et al. 1997). Furthermore, such ribozymes have been developed with the ability to target and modify mRNA transcripts for biochemical and therapeutic purposes (Sullenger and Cech 1994; Jones et al. 1996; Jones and Sullenger 1997; Phylactou et al. 1998; Kohler et al. 1999; Rogers et al. 2002; Byun et al. 2003; Ryu et al. 2003; Kastanos et al. 2004; Shin et al. 2004). For example, it was previously reported that a ribozyme derived from a Tetrahymena group I intron catalyzes a trans-splicing reaction (Sullenger and Cech 1994), in which the ribozyme binds an RNA substrate and replaces its defective $3^{\prime}$ exon sequence with a corrected form (carried by the ribozyme). The in vivo activity of this ribozyme has been

Reprint requests to: Stephen M. Testa, Department of Chemistry, University of Kentucky, Lexington, KY 40506, USA; e-mail: testa@uky.edu; fax: (859) 323-1069.

Article published online ahead of print. Article and publication date are at http://www.rnajournal.org/cgi/doi/10.1261/rna.2050505. demonstrated with various transcript targets (Sullenger and Cech 1994; Jones et al. 1996; Jones and Sullenger 1997; Phylactou et al. 1998; Kohler et al. 1999; Rogers et al. 2002; Byun et al. 2003; Ryu et al. 2003; Kastanos et al. 2004; Shin et al. 2004). This type of RNA modification has potential therapeutic application as it can be used to repair transcripts with mutations that lead to disease.

A complementary reaction to trans-splicing is the trans excision-splicing (TES) reaction (Bell et al. 2002). In this reaction, a group I intron-derived ribozyme from Pneumocystis carinii binds an RNA substrate and excises a targeted segment from within that substrate (Fig. 1). In vitro, this ribozyme has been used to excise inserts from 1 to 28 nucleotides in length and can be re-engineered to target nonnative sequences, including the flanking region of the trinucleotide repeat that causes myotonic dystrophy (Bell et al. 2002, 2004). The usefulness of these ribozymes, however, will be greatly enhanced by demonstrating in vivo reactivity.

In this report, we have designed a test system to assess the ability of the $P$. carinii ribozyme to catalyze the TES reaction in vivo. A single base insertion mutation was engineered into a green fluorescent protein (GFP) gene, which consequently destroys fluorescence of the resultant protein product. The TES ribozyme was then re-engineered to target this mutation, so a successful TES reaction reestablishes the reading frame of the protein in the GFP transcript (Fig. 2A), producing a fluorescing protein and thus a fluorescing cell culture. We 


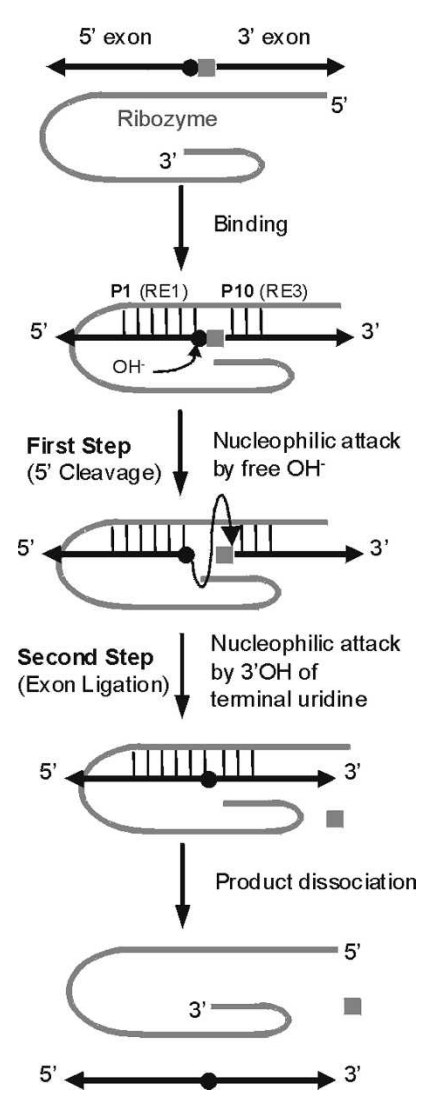

FIGURE 1. Ribozyme trans excision-splicing (TES) reaction. The TES ribozyme is shown as a gray line, the $5^{\prime}$ and $3^{\prime}$ exons are black lines, and the sequence targeted for excision (in this case, a single guanosine) is a gray box. The circle in the $5^{\prime}$ exon represents uridine. $\mathrm{P} 1$ and $\mathrm{P} 10$ are the helices that result from the ribozyme recognition elements RE1 and RE3 (respectively) base-pairing with the substrate.

now report that the ribozyme is able to catalyze the TES reaction in Escherichia coli. An average of $12 \%$ of the expected fluorescence was observed by using a construct with an elongated P10 helix of $5 \mathrm{bp}$, indicating an in vivo requirement for strong interactions between the ribozyme and the target transcript. Sequencing of isolated transcripts confirmed the ribozyme-mediated removal of the targeted nucleotide in at least $0.6 \%$ of the mutant transcripts. This represents the first report of a biomolecule (in this case a ribozyme) that can selectively excise a targeted nucleotide from within an mRNA transcript in vivo. This new class of biochemical tools makes possible a wide variety of new experimental strategies, perhaps including a new approach to molecular-based therapeutics.

\section{RESULTS}

\section{Design of the in vivo test system}

The design of the in vivo TES test system was based on work involving the trans-splicing ribozyme (Sullenger and Cech
1994; Jones et al. 1996; Jones and Sullenger 1997; Phylactou et al. 1998; Kohler et al. 1999; Rogers et al. 2002; Byun et al. 2003; Ryu et al. 2003; Kastanos et al. 2004; Shin et al. 2004), as well as design principles garnered through studies of the TES ribozyme in vitro (Bell et al. 2002, 2004). Our simplest TES reaction system involves the removal of a single nucleotide from a substrate, so we designed a test system in which we create a single base insertion mutation in the coding region of GFP (Fig. 2A). This mutation causes a deleterious shift in the reading frame of the transcribed RNA, which alters the amino acid sequence of the resultant protein product and creates a premature stop codon, resulting in a loss of fluorescence. We chose to insert a uridine $5^{\prime}$ to a guanosine in the RNA transcript (Fig. 2A). In the context of the TES reaction, this uridine will serve to define the $5^{\prime}$ splice site by forming the highly conserved $\mathrm{u}-\mathrm{G}$ wobble pair with a guanosine in the ribozyme (Barfod and Cech 1989; Doudna et al. 1989; Testa
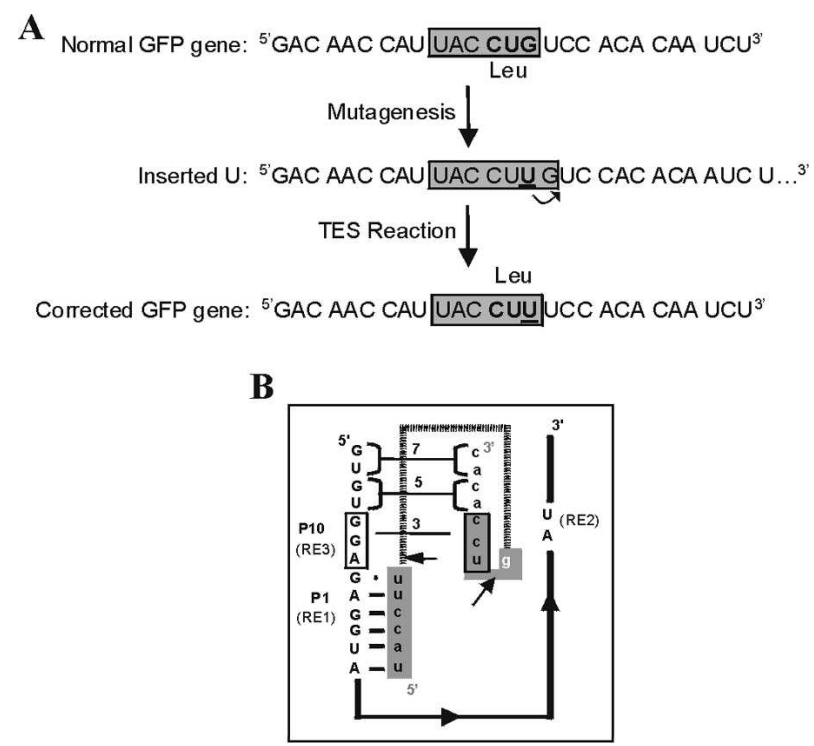

FIGURE 2. Design of the GFP target system $(A)$ and schematic of the two-step TES reaction utilizing the GFP target $(B)$. (A) The top line shows the sequence of interest of the GFP gene. The bold codon codes for leucine (Codon 201). The gray box is the sequence that base pairs with recognition element 1 (RE1) of the ribozyme. Site-directed mutagenesis results in the insertion of a uridine (underlined) $5^{\prime}$ to a guanosine in the mRNA transcript, which causes a frameshift and a premature stop codon (data not shown). The TES reaction removes the targeted guanosine and restores the correct reading frame of the GFP gene (bottom line). (B) Diagram of the recognition elements of the TES ribozyme base-pairing with the GFP target. The GFP rP3X ribozyme is in uppercase lettering, the GFP target is in lowercase lettering, and the guanosine to be excised is in white lettering. The ribozyme recognition elements RE1, RE2, and RE3 base pair with the substrate to form the P1, P9.0, and P10 helices, respectively. Note that the P9.0 helix is not utilized in this system. The sites of catalysis for the first step ( $5^{\prime}$ cleavage) and the second step (exon ligation) are shown with large bold arrows. The three-base P10 helix is boxed, with brackets indicating the extensions to five and seven bases. Note that the diagram only shows the recognition elements of the ribozyme. This ribozyme is the same as $\mathrm{rP}-8 / 4 \mathrm{x}$ (Testa et al. 1997), except for the sequences of RE1 and RE3 and the addition of a T7 terminator on the $3^{\prime}$ end. 
et al. 1997; Bell et al. 2002). The guanosine following the inserted uridine is equivalent to the $\omega$ position in the selfsplicing reaction (Price and Cech 1988; Burke 1989; Michel et al. 1989; Burke et al. 1990; van der Horst and Inoue 1993), so this $\omega \mathrm{G}$ is the base targeted for excision (Fig. 2B). After a successful TES reaction, the inserted uridine replaces the guanosine in the coding region, resulting in a silent mutation in the restored reading frame (Fig. 2A). Thus, the site for insertion was limited to those codons where replacement of guanosine by uridine results in the same amino acid when translated into protein. Codon 201, which codes for leucine, was an attractive target for insertion and, based on RNA structure prediction estimates, was accessible in the transcript. The insertion of a uridine and subsequent removal of a guanosine allow us to determine, via the sequence of isolated transcripts from our test reactions, that the fluorescent form of GFP results from a successful TES reaction.

Changes to modify the ribozyme were kept to a minimum for these initial in vivo studies and involved altering the recognition elements of the ribozyme, which are responsible for target identification (Fig. 2B). In all the test systems, the length of recognition element 1 (RE1) was maintained at six bases. Previous work on the TES ribozyme indicated that increasing the length of RE3 can be beneficial to the reaction (Bell et al. 2004), while work with the trans-splicing ribozyme showed a requirement for increased $3^{\prime}$ exon interactions for in vivo activity (Kohler et al. 1999; Rogers et al. 2002; Byun et al. 2003; Ryu et al. 2003; Kastanos et al. 2004; Shin et al. 2004). Thus, ribozymes were tested with RE3 lengths of three bases (native length), five bases, and seven bases. A third recognition element, RE2, is not utilized in TES reactions that remove a single nucleotide, so it was not modified. As a control for ribozyme activity, we also created mutant ribozyme constructs by deleting four bases from the guanosine binding site of the ribozyme. This mutation completely inactivates the ribozyme when tested in vitro (data not shown).

\section{The TES ribozyme is active in vivo}

First, the insertion mutation engineered into the GFP target was tested to ensure that it did indeed abolish fluorescence. The expected corrected form (with the G-to- $U$ transversion) was also tested for fluorescent activity. As seen in Figure 3, introducing the insertion mutation into the GFP gene abolishes GFP activity. The expected corrected form fluoresces, albeit at a level somewhat below that for the normal form of GFP, indicating the G-to-U transversion is not substantially disruptive to the translation of the GFP protein.

Next, the ribozymes engineered to target the mutation in GFP were tested for in vivo activity by pairing the ribozymes with the mutant form of GFP (Mut GFP). Targeted ribozymes (GFP rP3X) were tested with RE3 lengths of three, five, and seven bases. Increasing the length of RE3 increases the interaction between the ribozyme and the $3^{\prime}$ exon of the substrate, which helps prevent $3^{\prime}$ exon dissociation prior to

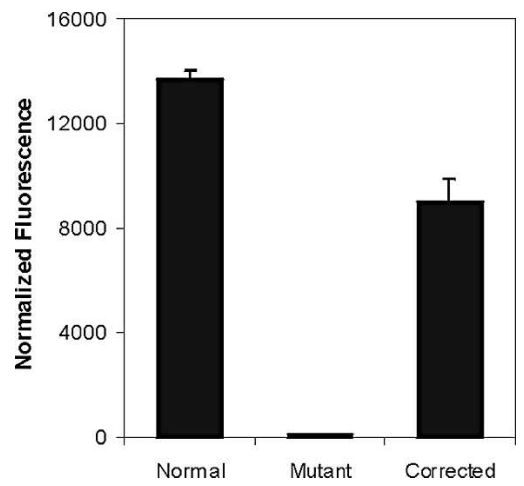

FIGURE 3. Comparison of the normalized GFP fluorescence levels for the normal, mutant, and corrected forms of GFP. All points utilize constructs with the nontargeted ribozyme and the GFP form listed on the graph. Normalized fluorescence was determined by correcting the raw fluorescence for differences in cell density (determined by the absorbance of the bulk culture at $600 \mathrm{~nm}$ ) and normalizing to noninduced controls. Each data point represents four independent assays, and the standard deviations are $<10 \%$.

the second reaction step and can improve TES product formation in vitro (Bell et al. 2004). The native form of the ribozyme (rP3X) served as a non-GFP-targeted control for ribozyme activity, while inactive forms of the ribozymes (containing a four-base deletion in the guanosine binding site) were used as negative controls. As seen in Figure 4, increasing the length of RE3 to five bases for the targeted ribozyme (GFP rP3X RE3=5) significantly increases the fluorescence over the inactive ribozyme controls, indicating TES reactivity. Note the level of fluorescence for the negative controls is not zero due to a low level of inherent fluorescence in the samples. We obtained an estimate of TES reactivity by comparing the data from the constructs with that of mutant GFP (Fig. 4) and the same ribozyme constructs with the

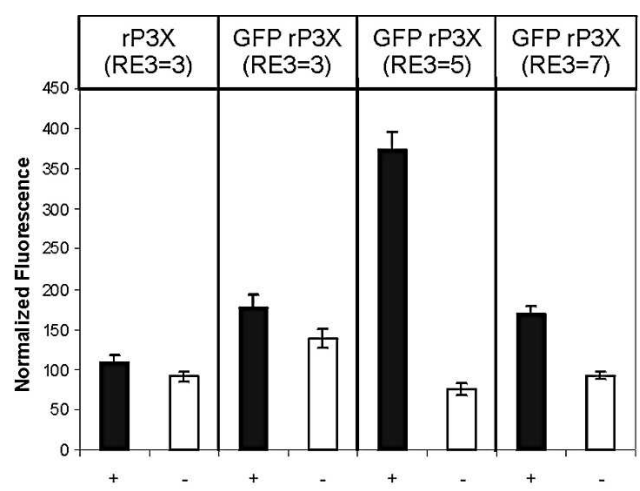

FIGURE 4. Normalized fluorescence data for constructs with mutant GFP paired with the TES ribozymes. All points utilize the mutant GFP target with the ribozyme listed above the graph. The plus symbols and solid bars indicate active ribozymes, while the minus symbols and open bars indicate the ribozymes have been inactivated by a deletion in the guanosine binding site. Normalized fluorescence was determined by correcting the raw fluorescence for differences in cell density (determined by the absorbance of the bulk culture at $600 \mathrm{~nm}$ ) and normalizing to noninduced controls. Each data point represents four independent assays, and the standard deviations are $<10 \%$. 
expected corrected form of GFP. For the corrected forms of GFP, normalized fluorescence values of 16,265.99 \pm 388.75 (ribozyme with an RE3 $=3$ ), $3105.83 \pm 1940.59$ (ribozyme with an RE3 = 5), and 5971.97 \pm 2272.05 (ribozyme with an RE3 $=7$ ) were obtained from at least three independent tests. Note the large standard deviations result from greater variability in reactions conducted in vivo when the RE3 length is increased from the native length of three nucleotides in constructs containing the corrected form of GFP. The estimated percentage of RNA repair was low for the targeted ribozymes with RE3 lengths of three nucleotides $(1.1 \pm 0.1 \%)$ and seven nucleotides $(3 \pm 1 \%)$, while utilizing a targeted ribozyme with an RE3 length of five nucleotides increased the estimated percentage of repair to $12 \pm 8 \%$.

\section{Sequence confirmation of in vivo TES reaction}

Total RNA was isolated from reactions involving three different constructs: the mutant GFP paired with the targeted ribozyme containing an RE3 $=5$ (the repair construct that showed the greatest increase in GFP activity), the mutant GFP paired with the inactive targeted ribozyme with an $\mathrm{RE} 3=5$ (a negative control), and the expected corrected form of GFP paired with the targeted ribozyme with an $\mathrm{RE} 3=5$ (a positive control to assess the insertion efficiency). The total RNA was subjected to RT-PCR to isolate the GFP transcript and the resulting RT-PCR products were ligated into a cloning vector and were assayed for their ability to produce fluorescent protein (Fig. 5). In the screening assays, 12 fluorescent colonies (out of $\sim 2000$ screened colonies) were obtained from products from two independent in vivo tests with the active repair construct, indicating the presence of corrected transcripts. Assays involving products from three

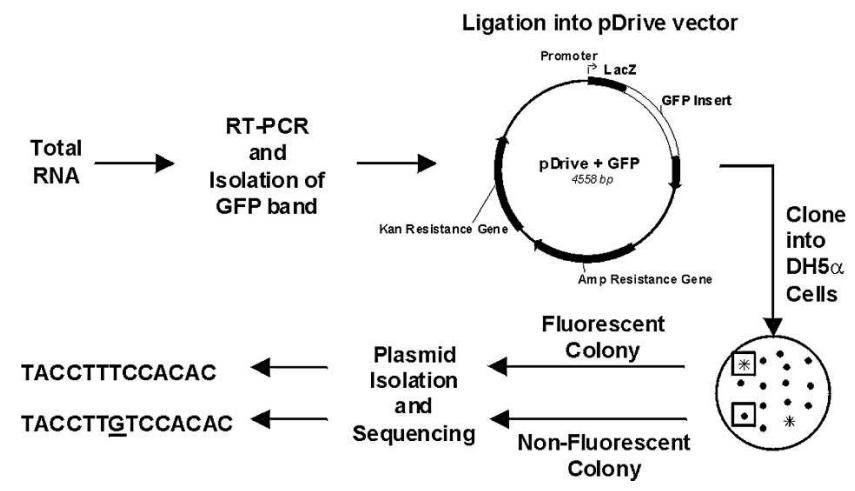

FIGURE 5. Schematic of the screening assay for isolation of corrected transcripts. Total RNA is isolated from cells that have undergone in vivo testing. The isolated RT-PCR products are ligated into the pDrive PCR cloning vector (Qiagen). Note that the RT-PCR products can be ligated into the vector in the forward direction (which puts the GFP gene in frame with the LacZ $\alpha$-peptide of the plasmid) or the reverse direction, with each direction assumed to be equally possible. Only products ligated in the forward direction will produce fluorescent GFP. The resulting plasmids are isolated and sequenced to confirm the expected sequence change in the corrected transcripts.

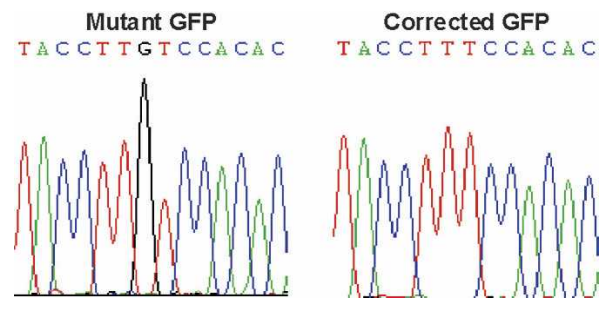

FIGURE 6. Sequencing confirmation of uncorrected GFP (left) and corrected GFP (right) transcripts. Total RNA isolated from in vivo tests involving the construct Mut GFP + GFP rP3X RE3 = 5 was utilized as the template for RT-PCR to isolate the GFP transcript.

independent in vivo tests with the mutant ribozyme construct produced no fluorescent colonies in $\sim 2000$ screened colonies, indicating that no TES reaction had occurred. The 12 fluorescent colonies from the active repair construct, one nonfluorescent colony from the active repair construct, and 18 colonies from the negative control construct were isolated, and the resulting plasmids were sequenced. All 12 sequences from the fluorescent colonies showed the removal of the targeted guanosine, resulting in the G-to- $\mathrm{U}$ transversion in the corrected transcript (Fig. 6). If the cell randomly fixed the transcript and produced fluorescent protein, the loss of a uridine would be anticipated to be as likely as the loss of a guanosine. As this loss of a uridine was not observed, the fluorescent protein was produced as a result of a successful TES reaction. There was also no evidence of a deletion that could serve as a compensatory mutation to restore fluorescence. The nonfluorescent colonies were confirmed to contain the unrepaired mutant GFP transcript (Fig. 6). Thus the removal of the guanosine was TES ribozyme mediated and the ribozyme is sequence specific for its target, without being deleterious to the cell. Based on this method, at least $0.6 \%$ of the mutant transcripts are corrected by the TES ribozyme.

\section{DISCUSSION}

The arsenal of molecular-based RNA tools is rapidly growing; however, the majority of these tools have focused on the destruction of the RNA message rather than the modification of the message (Sullenger and Gilboa 2002; Puerta-Fernandez et al. 2003; Scherer and Rossi 2003). Destroying the RNA message is useful for studying the effects of shutting off genes and can be useful in therapeutic applications to prevent the production of mutant proteins that lead to disease. On the other hand, modifying the message can restore the function to the transcript, thus reducing mutant protein and producing normal protein. With this in mind, we report the development of a catalytic RNA that can sequence-specifically target a single nucleotide within a cellular transcript in vivo and excise that targeted nucleotide from a transcript, with little or no apparent toxicity to the cell. Combined with the ease of adaptability, this provides for a wide variety of new in vivo experimental strategies. 
As biochemical tools, TES ribozymes could be used as inducible tools to modulate the production of proteins in vivo. As demonstrated in this report, TES ribozymes can be targeted to remove sequences to restore the reading frame of a transcript, thus producing active protein. The removal of sequences to shift the sequence out of frame is also possible, so inactive or mutant proteins could be produced in an inducible manner.

The applications of TES ribozymes could also include therapeutic applications. As a therapeutic agent, TES ribozymes could be used to remove insertion mutations and premature stop codons from transcripts, thus restoring the reading frame of the transcript for protein production. We have previously demonstrated the in vitro ability of a TES ribozyme to target a small model mimic of the triplet expansion implicated in myotonic dystrophy, and to excise from that mimic a short triplet expansion (Bell et al. 2002). Thus the repair of transcripts involved in triplet expansion diseases, such as muscular dystrophy and Huntington's disease, is another potential application for TES ribozymes in vivo.

As TES ribozymes recognize their targets initially and primarily through base-pairing, engineering the ribozymes to target new transcript regions simply requires changing the sequence of the recognition elements to base pair with the desired target. Other considerations for ribozyme targeting include reconstituting a u-G wobble pair at the $5^{\prime}$ splice site and a guanosine at the $3^{\prime}$ splice site as the last (or only) base of the sequence targeted for excision (Baum et al. 2005). Also note that previous work with this ribozyme demonstrated that segments larger than a single nucleotide can be excised in vitro (Bell et al. 2002), so we anticipate that larger regions could be excised in vivo.

\section{Comparing in vivo and in vitro TES results}

In our simplest test system, which involves the excision of a single, targeted nucleotide, the TES ribozyme is able to produce $70 \%$ product in vitro under optimized conditions of ribozyme excess and $10 \mathrm{mM} \mathrm{MgCl}_{2}$ (Bell et al. 2002). For the in vivo tests presented here, the ribozyme restores $\sim 12 \%$ of the fluorescence. This decrease in reactivity is not unexpected and is likely due to a number of factors, including a lower concentration of magnesium, a lower concentration of active ribozyme relative to the target substrate, and target accessibility. Nevertheless, TES ribozymes are adaptable to the cellular environment and are able to produce detectable amounts of repaired RNA.

\section{Comparison with trans-splicing and SMaRT}

TES ribozymes offer a different approach to RNA modification compared with the Tetrahymena-derived trans-splicing ribozyme (Sullenger and Cech 1994) and spliceosome-mediated RNA trans-splicing, or SMaRT (Puttaraju et al. 1999; Garcia-Blanco 2003). The transsplicing ribozyme replaces the $3^{\prime}$ exon of a targeted transcript with a new version of the exon, which is carried by the ribozyme. SMaRT utilizes the spliceosomes present in the cell to catalyze the splicing between an exon on the target transcript and an exon present in a pre-trans-splicing molecule (PTM). TES ribozymes are different in that they excise a targeted internal segment from the transcript.

TES ribozymes have some potential advantages over transsplicing ribozymes and SMaRT. TES ribozymes are not changed in the reaction, so they have the potential to be multipleturnover catalysts. The trans-splicing ribozyme splices its replacement exon into the target, making it single turnover. The PTM donates its replacement sequence during splicing, so it also is not a multiple turnover reaction. TES ribozymes can, in theory, be engineered to excise an internal segment anywhere on a transcript. In contrast, trans-splicing ribozymes are best suited to target mutations in the $3^{\prime}$ portion of a transcript, and SMaRT targets only exon/intron junctions. In addition, TES ribozymes do not carry replacement sequences, while trans-splicing ribozymes and PTMs do. Therefore, the size of the ribozyme-replacement exon construct or the PTM could become prohibitive for effective delivery and activity. Finally, in contrast to TES ribozymes and trans-splicing ribozymes, SMaRT can only be used in mammalian cells as it requires spliceosomes.

In E. coli, the level of activity restored by the trans-splicing ribozyme was $\sim 1 \%$ (Sullenger and Cech 1994). In our studies, we observe a higher level of repair in E. coli, as the level of activity restored by the TES ribozyme was $\sim 12 \%$. SMaRT is limited to mammalian cells, so we cannot accurately compare the levels of product formation to our E. coli results. That trans-splicing ribozymes (Jones et al. 1996; Jones and Sullenger 1997; Phylactou et al. 1998; Kohler et al. 1999; Rogers et al. 2002; Byun et al. 2003; Ryu et al. 2003; Kastanos et al. 2004; Shin et al. 2004) and SMaRT (Puttaraju et al. 1999; Liu et al. 2002; Chao et al. 2003) both work fairly well in mammalian cells (upward of 50\%) is encouraging, and we anticipate that TES ribozymes will also be active in mammalian systems. As is the case with trans-splicing (Jones and Sullenger 1997; Rogers et al. 2002; Byun et al. 2003; Shin et al. 2004) and SMaRT (Liu et al. 2002; Chao et al. 2003), we do not anticipate that TES modification will have to be $100 \%$ to have measurable effects as a biochemical tool. A low level of TES activity may produce a relevant amount of modified transcript for protein production.

Note that the challenges we face in improving the TES reaction in vivo and adapting TES ribozymes to a mammalian system are the same challenges faced by others developing ribozymes in mammalian systems (Sullenger and Gilboa 2002; Long et al. 2003; Scherer and Rossi 2003). Common issues in E. coli and mammalian cells include increasing the level of ribozyme activity, improving ribozyme specificity, assessing target accessibility, and effectively delivering the ribozyme. Work is ongoing to address these issues and is encouraging (Sullenger and Gilboa 2002; Long et al. 2003; Puerta-Fernandez et al. 2003; Scherer and Rossi 2003). In conclusion, this study demonstrates the potential of TES 
ribozymes as new tools for the in vivo sequence-specific modification of RNA transcripts.

\section{MATERIALS AND METHODS}

\section{Oligonucleotide synthesis and preparation}

DNA oligonucleotides were purchased from Integrated DNA Technologies. Oligonucleotides for the insertion of the T7 terminator sequence were $5^{\prime}$ phosphorylated and PAGE purified by the company. Oligonucleotides for site-directed mutagenesis and RT-PCR were used without further purification.

\section{Plasmid construction}

The $P$. carinii ribozyme precursor plasmid was constructed as previously described (Testa et al. 1997). To stop transcription of the ribozyme in vivo, a T7 terminator sequence (Studier et al. 1990) was inserted immediately $3^{\prime}$ to the ribozyme sequence by
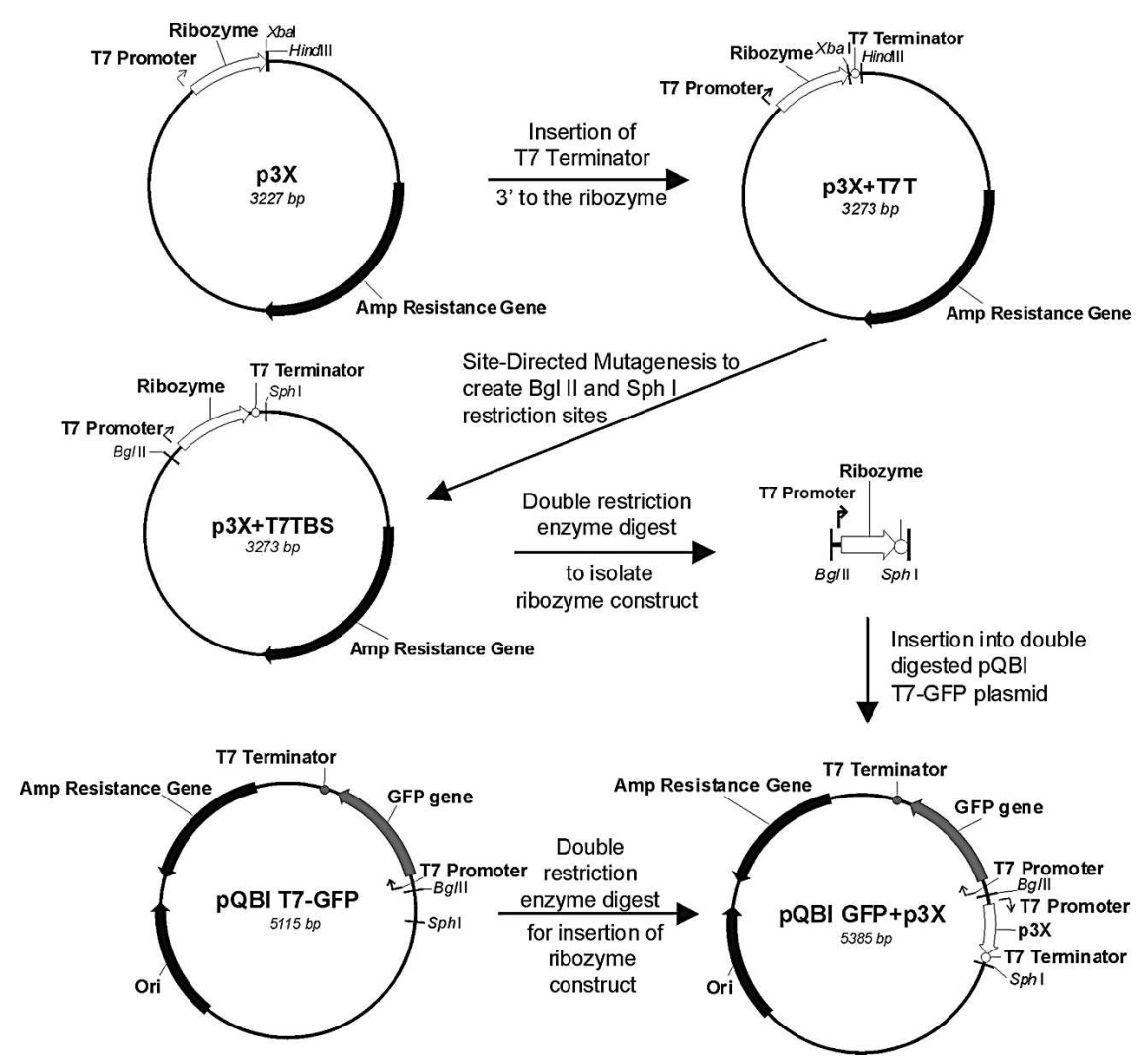

FIGURE 7. Construction of the in vivo TES test plasmid. The original TES ribozyme plasmid (P3X) was modified by the insertion of a linker containing a T7 terminator sequence $3^{\prime}$ to the ribozyme sequence to create $\mathrm{P} 3 \mathrm{X}+\mathrm{T} 7 \mathrm{~T}$. Site-directed mutagenesis was used to create a unique BglII site $5^{\prime}$ to the T7 promoter of the ribozyme and a unique SphI site $3^{\prime}$ to the T7 terminator of the ribozyme (P3X+T7TBS). The ribozyme construct was isolated from the plasmid by a double restriction enzyme digest with BgIII and SphI. The GFP-containing plasmid, pQBI T7-GFP (Qbiogene), was prepared for insertion of the ribozyme construct by a double enzyme digest with BglII and SphI. The ribozyme construct was ligated into the cut pQBI T7-GFP plasmid to create $\mathrm{pQBI}$ GFP + P3X. Modifications to the GFP gene to create the mutant and corrected forms and modifications to the ribozyme to change the recognition elements and to create the inactive forms were all created via site-directed mutagenesis. using the XbaI and HindIII sites present in the P3X plasmid (Fig. 7). The ligation product was used to transform E. coli DH5 $\alpha$ competent cells (Invitrogen). The resulting plasmid $(\mathrm{P} 3 \mathrm{X}+\mathrm{T} 7 \mathrm{~T})$ was purified by using a QIAprep Spin Miniprep kit (Qiagen) and sequenced for confirmation (ACGT, Inc.).

Two unique restriction sites, Bglll and Sphl, were engineered into ribozyme construct (Fig. 7). The plasmid, P3X + T7TBS, was created by two successive rounds of site-directed mutagenesis. The following primer pair was used to create a BglII restriction site upstream of the T7 promoter: $5^{\prime}$-GGAAACAGATCTGACATGATTACGAATTT GG-3' and $5^{\prime}$-CCAAATTCGTAATCATGTCAGATCTGTTTCC- $3^{\prime}$. A SphI restriction site was created downstream of the T7 terminator with the following primer pair: 5'-GCTTACTAGTGATGCATGC TCTATAGTGTCACC-3' and 5'-GGTGACACTATAGAGCATGC ATCACTAGTAAGC- $3^{\prime}$. The site-directed mutagenesis reactions were conducted as previously described (Bell et al. 2002), with the changes that follow. The reaction mixtures were subjected to denaturation for $30 \mathrm{sec}$ at $95^{\circ} \mathrm{C}$, followed by 15 temperature cycles of $30 \mathrm{sec}$ at $95^{\circ} \mathrm{C}, 2 \mathrm{~min}$ at $55^{\circ} \mathrm{C}$, and 6 min at $68^{\circ} \mathrm{C}$. The parental plasmids were digested with $20 \mathrm{U}$ of DpnI (New England Biolabs) in $4.2 \mu \mathrm{L}$ of manufacturer's buffer for at least $1 \mathrm{~h}$ at $37^{\circ} \mathrm{C}$. A 3- $\mu \mathrm{L}$ aliquot of this digest was then used to transform E. coli DH5 $\alpha$ competent cells (Invitrogen). The resultant plasmids were purified as above and were sequenced for confirmation (Davis Sequencing).

The plasmid containing the GFP target, pQBI T7-GFP, was purchased from Qbiogene. The base plasmid for testing, pQBI GFP + P3X, was created by inserting the ribozyme construct into the $\mathrm{pQBI}$ T7-GFP plasmid by using the BgIII and the SphI restriction sites present in the pQBI T7-GFP plasmid (Fig. 7). The ribozyme construct was isolated by digesting the P3X + T7TBS plasmid with BglII and SphI. The ligation product was used to transform E. coli $\mathrm{DH} 5 \alpha$ competent cells (Invitrogen). The resulting plasmid was purified and sequenced as above.

Test plasmids were generated via sitedirected mutagenesis of the base plasmid pQBI GFP + P3X. The following primers were used to modify the GFP gene to contain a single base insertion mutation to destroy GFP fluorescence (Mut GFP): 5'-GCA GATTGTGTGGACAAGGTAATGGTTGT CTGG- $3^{\prime}$ and $5^{\prime}$-CCAGACAACCATTACC TTGTCCACACAATCTGC- $3^{\prime}$. The underlined base represents the insertion mutation. The corrected form of GFP expected from a successful TES reaction (Corr GFP) was created with the following primers: 5'-GCA GATTGTGTGGAAAGGTAATGGTTGTC TGG- $3^{\prime}$ and $5^{\prime}$-CCAGACAACCATTACCT TTCCACACAATCTGC- $3^{\prime}$. The underlined base represents the silent mutation expected 
from removal of the targeted guanosine by the TES ribozyme. These mutations alter codon 201 of the GFP gene. Site-directed mutagenesis was performed as above, with the following modifications. The reaction mixtures were subjected to denaturation for $30 \mathrm{sec}$ at $95^{\circ} \mathrm{C}$, followed by 15 or 18 temperature cycles of $30 \mathrm{sec}$ at $95^{\circ} \mathrm{C}, 2 \mathrm{~min}$ at $50^{\circ} \mathrm{C}$ or $55^{\circ} \mathrm{C}$, and 6 or $8 \mathrm{~min}$ at $68^{\circ} \mathrm{C}$, depending on the construct. Resulting plasmids were purified and sequenced as above.

The ribozyme portion of the test plasmid was modified in the following ways by using site-directed mutagenesis. First, the recognition elements of the ribozyme (RE1 and RE3) were modified to recognize the GFP target by two successive rounds of site-directed mutagenesis. A third recognition element, RE2, is not utilized in TES reactions involving the removal of a single nucleotide, so it was not modified. The primers for the first round were as follows: $5^{\prime}$-CGACT CACTATAGAGGGGGTAGAAAGCGGC- $3^{\prime}$ and $5^{\prime}$-GCCGCTTTC TACCCCCTCTATAGTGAGTCG- $3^{\prime}$. The second round of changes was made with the following primers: $5^{\prime}$-CGACTCACTATAGGA GAGGTAGAAAGCGGC-3' ${ }^{\prime}$ and $5^{\prime}$-GCCGCTTTCTACCTCTCCTA TÄGTGAGTCG-3'. The length of RE3 was increased to five bases by using the following primers: $5^{\prime}$-CGACTCACTATAGTGGAGAGG TAGAAAGCGGC- $3^{\prime}$ and $5^{\prime}$-GCCGCTTTCTACCTCTCCACTATAG TGAGTCG-3' . An RE3 containing seven bases was created by using the following primer pair: $5^{\prime}$-CGACTCACTATAGTGTGGAGAGGTAG AAAGCGGC- $3^{\prime}$ and $5^{\prime}$-GCCGCTTTCTACCTCTCCACACTATAGT GAGTCG-3' . A mutant form of the ribozyme was created by deleting four bases (bases 250-253) from the guanosine binding site of the ribozyme (Testa et al. 1997). Site-directed mutagenesis reactions were performed as above, and the resulting plasmids were sequenced for confirmation (Davis Sequencing).

\section{Preparing competent JM109(DE3) and transforming competent JM109(DE3)}

In vivo testing of the TES ribozyme was conducted in $E$. coli strain JM109(DE3) (Promega). Competent JM109(DE3) cells were prepared by using the rubidium chloride protocol reported by Promega (Doyle 1996). Following a $1 \mathrm{~h}$ incubation on ice, the competent cells were transformed with the test plasmids by using a modified version of the procedure outlined by Promega (Doyle 1996). One hundred microliters of competent cells was used in each transformation reaction. Approximately $10 \mathrm{ng}$ of test plasmid was added to the competent cells, and the tubes were incubated for $30 \mathrm{~min}$ on ice. The tubes were then heated for $50 \mathrm{sec}$ at $44^{\circ} \mathrm{C}$ and placed immediately back on ice for $2 \mathrm{~min} ; 1.5 \mathrm{~mL}$ of LB media was then added to each tube, and the tubes were incubated at $37^{\circ} \mathrm{C}$ with shaking at $225 \mathrm{rpm}$ for $45 \mathrm{~min}$. After incubation, the transformations were plated on LB plates containing ampicillin, and the plates were incubated overnight at $37^{\circ} \mathrm{C}$.

\section{In vivo TES reactions}

The colonies resulting from transformation of the competent JM109(DE3) cells were used for in vivo testing of the TES ribozymes. Colonies were isolated and grown up in liquid culture overnight. The following day, the overnight cultures were used to inoculate fresh LB media without antibiotic to create 1:20 dilution cultures. The dilution cultures were incubated at $37^{\circ} \mathrm{C}$ with shaking at $225 \mathrm{rpm}$ for $3 \mathrm{~h}$ to allow growth to reach log phase $\left(\mathrm{A}_{600}>0.4\right)$. After $3 \mathrm{~h}$, aliquots were removed from each culture for cell density analysis $(500 \mu \mathrm{L})$ and fluorescence analysis $(1 \mathrm{~mL})$. The rest of the dilution culture was then subdivided. The inducer, isopropylthiogalactoside (IPTG), was added to one of the cultures to a final concentration of $\sim 1.3 \mathrm{mM}$, while the other culture served as a noninduced control. Cultures were incubated at $37^{\circ} \mathrm{C}$ with shaking at $225 \mathrm{rpm}$ for $5 \mathrm{~h}$. After $5 \mathrm{~h}$, aliquots were removed for cell density analysis $(500 \mu \mathrm{L})$, GFP fluorescence analysis $(1 \mathrm{~mL})$, and, for select cultures, total RNA isolation $(1 \mathrm{~mL})$.

\section{Analysis}

Cell density was determined by measuring the absorbance of the culture at $600 \mathrm{~nm}\left(\mathrm{~A}_{600}\right)$. For GFP fluorescence analysis, the culture aliquots were centrifuged to pellet the cells. The media was removed, and the cell pellets were resuspended in $300 \mu \mathrm{L}$ of phosphate buffered saline (PBS) at $\mathrm{pH}$ 7.4. The resuspended pellets were then loaded into a FluoroNunc MaxiSorp 96-well plate (Nalge Nunc International). GFP fluorescence was measured in a CytoFluor (PerSeptive Biosystems), using a $485 \pm 20 \mathrm{~nm}$ filter for excitation and a $508 \pm 20 \mathrm{~nm}$ filter for emission, as the excitation wavelength for this form of GFP is $474 \mathrm{~nm}$ and the emission wavelength is $509 \mathrm{~nm}$. The raw fluorescence measurements were corrected for differences in cell culture growth by dividing the raw fluorescence values by the $A_{600}$ readings. These corrected fluorescence values were then normalized to the noninduced values to correct for "leaky" T7 RNA polymerase activity by subtracting the corrected fluorescence for the noninduced culture from the corrected fluorescence for the induced culture. The reported normalized fluorescence values are the result of at least three independent assays. Note that omitting the corrections for differences in cell density does not significantly affect the trends obtained (data not shown). Error limits for the estimated percentage of RNA repair were calculated as for multiplicative expressions (Miller and Miller 2000).

\section{Total RNA isolation}

Total RNA was isolated by using $1 \mathrm{~mL}$ aliquots from designated cultures using the Ambion RiboPure-Bacteria Kit (Ambion, Inc.) and was treated with RQ1 RNase-Free DNase (Promega) to completely remove the DNA prior to RT-PCR. RNA was isolated from the DNase reaction mixture by acid phenol ( $\mathrm{pH} 4.3$ ) extraction, followed by phenol/chloroform extraction and ethanol precipitation.

\section{RT-PCR reactions}

The GFP transcripts were amplified from the total RNA by RT-PCR using the following primers: $5^{\prime}$-GTTGTACAGTTCATC CATGCC- $3^{\prime}$ and $5^{\prime}$-GGAGAAGAACTCTTCACTGG-3'. RT-PCR reactions were performed by using the Access RT-PCR System (Promega) and consisted of $50 \mu \mathrm{L}$ reactions containing $\sim 1 \mu \mathrm{g}$ total RNA, $1 \mathrm{mM} \mathrm{MgSO}_{4}$, 45 pmol of each primer, $0.2 \mathrm{mM}$ dNTPs, 5 U AMV reverse transcriptase, and 5 U Tfl DNA polymerase in the provided reaction buffer. PCR reactions were performed as a control to ensure the DNA had been removed from the samples. The reactions were subjected to $45 \mathrm{~min}$ at $45^{\circ} \mathrm{C}$ for first-strand cDNA synthesis, followed by 2 min at $94^{\circ} \mathrm{C}$ to inactivate the AMV reverse transcriptase. The reactions then underwent 40 temperature cycles consisting $30 \mathrm{sec}$ at $94^{\circ} \mathrm{C}, 1 \mathrm{~min}$ at $54^{\circ} \mathrm{C}$, 
and $2 \mathrm{~min}$ at $68^{\circ} \mathrm{C}$. After cycling was complete, the reactions underwent a final extension cycle for $10 \mathrm{~min}$ at $68^{\circ} \mathrm{C}$. The RT-PCR products were separated on a $2 \%$ agarose gel, and the GFP band was excised. The band was extracted from the gel matrix by using a QIAquick Gel Extraction Kit (Qiagen). The gel-purified products were ethanol precipitated twice prior to use in ligation reactions.

\section{Assay for corrected transcripts}

The TES reaction removes a targeted sequence from within an RNA, so the $5^{\prime}$ and $3^{\prime}$ ends are the same in the mutant and corrected forms. Moreover, in our simple system, the difference between the mutant GFP and the corrected GFP is a single base. Thus, the product cannot trivially be selectively amplified by RT-PCR. Therefore, we designed an assay to analyze the pool of transcripts after the reaction for those that are corrected and thus produce fluorescent protein (Fig. 5). The GFP RT-PCR products were ligated into the pDrive cloning vector by using the QIAGEN PCR Cloning kit (Qiagen). Ligation reactions consisted of $50 \mathrm{ng}$ pDrive cloning vector, $200 \mathrm{ng}$ of RT-PCR product, $1 \mu \mathrm{L} 50 \%$ PEG $(5 \%$ final $\mathrm{w} / \mathrm{v}$ ), and $2 \times$ Ligation Master Mix (provided with the kit) in a final volume of $10 \mu \mathrm{L}$. Ligations proceeded for $1.5 \mathrm{~h}$ at $4^{\circ} \mathrm{C}$. Following ligation, a $3-\mu \mathrm{L}$ aliquot of the ligation reaction was used to transform E. coli DH5 $\alpha$ competent cells (Invitrogen). Transformations were plated on LB media plates containing kanamycin, and the plates were incubated overnight at $37^{\circ} \mathrm{C}$. The following day, the plates were placed at $4^{\circ} \mathrm{C}$ for at least $4 \mathrm{~h}$ to enhance GFP fluorescence for visualization. Those colonies containing the corrected GFP products will produce fluorescent GFP, which can be visualized on the plate. After incubation at $4^{\circ} \mathrm{C}$, the plates were exposed to UV light, and colonies showing GFP activity were isolated. The resulting plasmids were isolated by using a QIAprep Spin Miniprep kit (Qiagen) and sequenced for confirmation (Davis Sequencing).

\section{ACKNOWLEDGMENTS}

We thank Rashada Alexander, Dustin Lafferty, and Joy Sinha for technical assistance and members of the Testa lab for helpful discussion. We also thank Dr. Sylvia Daunert (Department of Chemistry, University of Kentucky) and members of the Daunert laboratory for use of the CytoFluor. This work was supported by the Muscular Dystrophy Association and the DOD Breast Cancer Research Program DAMD17-03-1-0329.

Received January 10, 2005; accepted March 2, 2005.

\section{REFERENCES}

Barfod, E.T. and Cech, T.R. 1989. The conserved U.G pair in the $5^{\prime}$ splice site duplex of a group I intron is required in the first but not the second step of self-splicing. Mol. Cell. Biol. 9: 3657-3666.

Baum, D.A., Sinha, J., and Testa, S.M. 2005. Molecular recognition in a trans excision-splicing ribozyme: Non-Watson-Crick base pairs at the $5^{\prime}$ splice site and $\omega \mathrm{G}$ at the $3^{\prime}$ splice site can play a role in determining the binding register of reaction substrates. Biochemistry 44: 1067-1077.
Bell, M.A., Johnson, A.K., and Testa, S.M. 2002. Ribozyme-catalyzed excision of targeted sequences from within RNAs. Biochemistry 41: 15327-15333.

Bell, M.A., Sinha, J., Johnson, A.K., and Testa, S.M. 2004. Enhancing the second step of the trans excision-splicing reaction of a group I ribozyme by exploiting P9.0 and P10 for intermolecular recognition. Biochemistry 43: 4323-4331.

Burke, J.M. 1989. Selection of the $3^{\prime}$-splice site in group I introns. FEBS Lett. 250: 129-133.

Burke, J.M., Esherick, J.S., Burfeind, W.R., and King, J.L. 1990. A $3^{\prime}$ splice site-binding sequence in the catalytic core of a group I intron. Nature 344: 80-82.

Byun, J., Lan, N., Long, M., and Sullenger, B.A. 2003. Efficient and specific repair of sickle $\beta$-globin RNA by trans-splicing ribozymes. RNA 9: 1254-1263.

Cech, T.R. 1990. Self-splicing of group I introns. Annu. Rev. Biochem. 59: 543-568.

Chao, H., Mansfield, S.G., Bartel, R.C., Hiriyanna, S., Mitchell, L.G., Garcia-Blanco, M.A., and Walsh, C.E. 2003. Phenotype correction of hemophilia A mice by spliceosome-mediated RNA trans-splicing. Nat. Med. 9: 1015-1019.

Doudna, J.A., Cormack, B.P., and Szostak, J.W. 1989. RNA structure, not sequence, determines the $5^{\prime}$ splice-site specificity of a group I intron. Proc. Natl. Acad. Sci. 86: 7402-7406.

Doyle, K. 1996. Promega protocols and applications guide. Promega Corporation, Madison, WI.

Garcia-Blanco, M.A. 2003. Mending the message. Nat. Biotechnol. 21: $1448-1449$.

Jones, J.T. and Sullenger, B.A. 1997. Evaluating and enhancing ribozyme reaction efficiency in mammalian cells. Nat. Biotechnol. 15: 902-905.

Jones, J.T., Lee, S.W., and Sullenger, B.A. 1996. Tagging ribozyme reaction sites to follow trans-splicing in mammalian cells. Nat. Med. 2: 643-648.

Kastanos, E., Hjiantoniou, E., and Phylactou, L.A. 2004. Restoration of protein synthesis in pancreatic cancer cells by trans-splicing ribozymes. Biochem. Biophys. Res. Commun. 322: 930-934.

Kohler, U., Ayre, B.G., Goodman, H.M., and Haseloff, J. 1999. Transsplicing ribozymes for targeted gene delivery. J. Mol. Biol. 285: 1935-1950.

Kruger, K., Grabowski, P.J., Zaug, A.J., Sands, J., Gottschling, D.E., and Cech, T.R. 1982. Self-splicing RNA: Autoexcision and autocyclization of the ribosomal RNA intervening sequence of Tetrahymena. Cell 31: 147-157.

Liu, X., Jiang, Q., Mansfield, S.G., Puttaraju, M., Zhang, Y., Zhou, W., Cohn, J.A., Garcia-Blanco, M.A., Mitchell, L.G., and Engelhardt, J.F. 2002. Partial correction of endogenous $\Delta$ F508 CFTR in human cystic fibrosis airway epithelia by spliceosome-mediated RNA trans-splicing. Nat. Biotechnol. 20: 47-52.

Long, M.B., Jones III, J.P., Sullenger, B.A., and Byun, J. 2003. Ribozymemediated revision of RNA and DNA. J. Clin. Invest. 112: 312-318.

Michel, F., Hanna, M., Green, R., Bartel, D.P., and Szostak, J.W. 1989. The guanosine binding site of the Tetrahymena ribozyme. Nature 342: 391-395.

Miller, J.N. and Miller, J.C. 2000. Statistics of repeated measurements: Propagation of random errors. In Statistics and chemometrics for analytical chemistry, pg. 36. Prentice Hall, Harlow, England.

Phylactou, L.A., Darrah, C., and Wood, M.J. 1998. Ribozyme-mediated trans-splicing of a trinucleotide repeat. Nat. Genet. 18: 378-381.

Price, J.V. and Cech, T.R. 1988. Determinants of the $3^{\prime}$ splice site for selfsplicing of the Tetrahymena pre-rRNA. Genes \& Dev. 2: 1439-1447.

Puerta-Fernandez, E., Romero-Lopez, C., Barroso-delJesus, A., and Berzal-Herranz, A. 2003. Ribozymes: Recent advances in the development of RNA tools. FEMS Microbiol. Rev. 27: 75-97.

Puttaraju, M., Jamison, S.F., Mansfield, S.G., Garcia-Blanco, M.A., and Mitchell, L.G. 1999. Spliceosome-mediated RNA transsplicing as a tool for gene therapy. Nat. Biotechnol. 17: 246-252.

Rogers, C.S., Vanoye, C.G., Sullenger, B.A., and George Jr., A.L. 2002. Functional repair of a mutant chloride channel using a transsplicing ribozyme. J. Clin. Invest. 110: 1783-1789. 
Ryu, K.J., Kim, J.H., and Lee, S.W. 2003. Ribozyme-mediated selective induction of new gene activity in hepatitis $\mathrm{C}$ virus internal ribosome entry site-expressing cells by targeted trans-splicing. Mol. Ther. 7: 386-395.

Sargueil, B. and Tanner, N.K. 1993. A shortened form of the Tetrahymena thermophila group I intron can catalyze the complete splicing reaction in trans. J. Mol. Biol. 233: 629-643.

Scherer, L.J. and Rossi, J.J. 2003. Approaches for the sequence-specific knockdown of mRNA. Nat. Biotechnol. 21: 1457-1465.

Shin, K.S., Sullenger, B.A., and Lee, S.W. 2004. Ribozyme-mediated induction of apoptosis in human cancer cells by targeted repair of mutant p53 RNA. Mol. Ther. 10: 365-372.

Studier, F.W., Rosenberg, A.H., Dunn, J.J., and Dubendorff, J.W. 1990. Use of T7 RNA polymerase to direct expression of cloned genes. Methods Enzymol. 185: 60-89.
Sullenger, B.A. and Cech, T.R. 1994. Ribozyme-mediated repair of defective mRNA by targeted, trans-splicing. Nature 371: 619-622.

Sullenger, B.A. and Gilboa, E. 2002. Emerging clinical applications of RNA. Nature 418: 252-258.

Testa, S.M., Haidaris, C.G., Gigliotti, F., and Turner, D.H. 1997. A Pneumocystis carinii group I intron ribozyme that does not require $2^{\prime} \mathrm{OH}$ groups on its $5^{\prime}$ exon mimic for binding to the catalytic core. Biochemistry 36: 15303-15314.

van der Horst, G. and Inoue, T. 1993. Requirements of a group I intron for reactions at the $3^{\prime}$ splice site. J. Mol. Biol. 229: 685-694.

Zaug, A.J. and Cech, T.R. 1986. The intervening sequence RNA of Tetrahymena is an enzyme. Science 231: 470-475.

Zaug, A.J., Been, M.D., and Cech, T.R. 1986. The Tetrahymena ribozyme acts like an RNA restriction endonuclease. Nature 324: 429-433. 

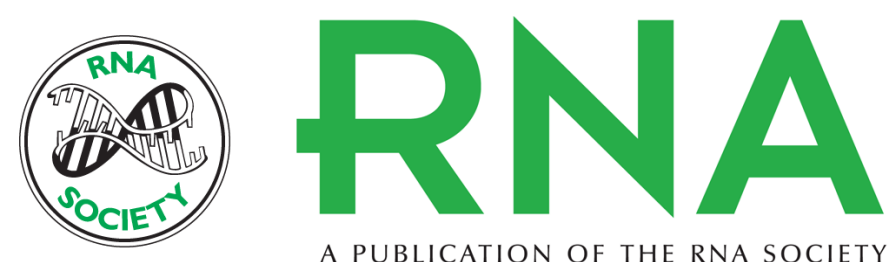

A PUBLICATION OF THE RNA SOCIETY

\section{In vivo excision of a single targeted nucleotide from an mRNA by a trans excision-splicing ribozyme}

DANA A. BAUM and STEPHEN M. TESTA

RNA 2005 11: 897-905

References This article cites 35 articles, 5 of which can be accessed free at:

http://rnajournal.cshlp.org/content/11/6/897.full.html\#ref-list-1

License

Email Alerting Receive free email alerts when new articles cite this article - sign up in the box at the Service top right corner of the article or click here. 